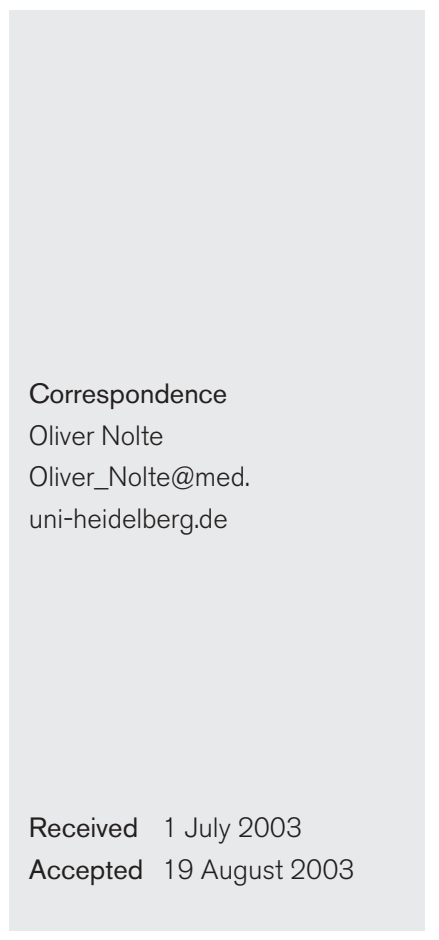

\title{
Description of new mutations in the rpoB gene in rifampicin-resistant Neisseria meningitidis selected in vitro in a stepwise manner
}

\author{
Oliver Nolte, ${ }^{1}$ Matthias Müller, ${ }^{1}$ Stephan Reitz, ${ }^{1}$ Sandra Ledig, ${ }^{1}$ \\ Ingrid Ehrhard ${ }^{2}$ and Hans-Günther Sonntag ${ }^{1}$ \\ ${ }^{1}$ Hygiene Institute, Dept of Hygiene and Medical Microbiology, University of Heidelberg, Im \\ Neuenheimer Feld 324, D-69120 Heidelberg, Germany \\ ${ }^{2}$ Landesuntersuchungsanstalt für das Gesundheits- und Veterinärwesen (LUA) Sachsen, Standort \\ Dresden, Abt. Med. Mikrobiologie und Hygiene, Haus Jägerstr. 10, D-01099 Dresden, Germany
}

\begin{abstract}
Fourteen meningococcal strains were selected towards rifampicin resistance in a stepwise manner in vitro; final MICs were between 8 and $>256 \mu \mathrm{g} \mathrm{ml}^{-1}$. Sequence analysis of a $295 \mathrm{bp}$ subgenic fragment of the RNA polymerase $\beta$-subunit $(r p o B)$ gene from the original and the fully resistant strains revealed that, with one exception, the strain pairs differed by just one position in the deduced amino acid sequence. Transformation of a PCR-amplified subgenic $r p o B$ fragment harbouring the mutated site into a susceptible strain demonstrated the resistance-conferring mechanism.
\end{abstract}

\section{INTRODUCTION}

Many reports have described single point mutations leading to rifampicin resistance in a variety of micro-organisms, for instance, in Escherichia coli (Jin \& Gross, 1988), Mycobacterium tuberculosis (Telenti et al., 1993) and Staphylococcus aureus (Aubry-Damon et al., 1998). The mutations described provided the first evidence that mutations within a defined subgenic segment of the $\beta$-subunit of DNA-dependent RNA polymerase (encoded by $r p o B$ ) may be the mechanism that causes rifampicin resistance. A couple of different point mutations in a subgenic $r p o B$ fragment have also been described for Neisseria meningitidis (Carter et al., 1994; Nolte, 1997; Stefanelli et al., 2001). The hitherto-described point mutations were considered to be responsible for the single-step development of resistance. Additional mechanisms such as mutations in membrane efflux pumps, which were originally described for Neisseria gonorrhoeae resistant to hydrophobic agents (Hagman et al., 1995; Pan \& Spratt, 1994), have been discussed as a reason for high-level resistance (Abadi et al., 1996). However, nothing is currently known about the possible stepwise acquisition of rifampicin resistance, neither whether this can indeed happen nor which molecular event might be the mechanism. The current work was therefore intended to select primary susceptible meningococci towards rifampicin resistance in vitro in a stepwise manner. The basic idea behind our approach was that, if we selected single colonies that appeared to be less susceptible to rifampicin than the majority of the respective plate culture on an E-test, we should be able to force the meningococci to stepwise higher MICs. Subsequent sequencing of the sub- genic $r p o B$ fragment should lead to the identification of point mutations that confer resistance by means of a cumulative mechanism.

\section{METHODS}

Fourteen N. meningitidis strains (Table 1), which were originally isolated from routine diagnostic material sent to the German Reference Centre for Meningococci (at the time that this study was initiated, the Centre was located at Hygiene-Institute, University of Heidelberg; now located at the Hygiene-Institute, University of Würzburg), were used. In order to select stepwise for rifampicin resistance, E-tests were performed using rifampicin E-tests strips (AB Biodisk; Solna) according to the recommendations of the manufacturer. Following $18 \mathrm{~h}$ incubation, those colonies growing slightly into the inhibition ellipse were subcultured and used for a subsequent E-test. This procedure was repeated until a strain was found to be resistant. The criterion for resistance was an MIC $\geqslant 4 \mu \mathrm{g} \mathrm{ml}^{-1}$, as recommended in the NCCLS guidelines for organisms other than Haemophilus ssp., N. gonorrhoeae and streptococci.

The DNA of each strain was subjected to PCR in order to amplify a 790 bp subgenic rpoB fragment (fragment B in Fig. 1) as described previously (Nolte, 1997). Amplification products were purified and analysed by cycle sequencing. A SequiTherm Excel II Long-Read DNA sequencing kit ALF (Epicentre Technologies) and the sequencing primers NmB9F and NmB24R (sequences given in Table 2; positions on $r p o B$ are shown in Fig. 1) were used for automated sequencing on an ALFexpress sequencer (Amersham Pharmacia Biotech).

For the transformation experiments, an amplicon of $1352 \mathrm{bp}$ (fragment A in Fig. 1) of $r p o B$, covering the $\operatorname{Ser}^{600}$ mutation, was amplified 
Table 1. Meningococcal strain pairs used in this work

Amino acid positions refer to the position in the complete RpoB protein. The phenotype gives the serological formula of the original strains.

\begin{tabular}{|c|c|c|c|c|c|c|}
\hline \multicolumn{2}{|c|}{ Original isolate } & \multicolumn{2}{|c|}{ Resistant isolate } & \multirow[t]{2}{*}{ Passages $(n)^{\star}$} & \multirow{2}{*}{$\begin{array}{l}\text { Amino acid } \\
\text { substitution(s) }\end{array}$} & \multirow[t]{2}{*}{ Phenotype } \\
\hline Strain & $\operatorname{MIC}\left(\mu \mathrm{g} \mathrm{ml}^{-1}\right)$ & Strain & $\operatorname{MIC}\left(\mu \mathrm{g} \mathrm{ml}^{-1}\right)$ & & & \\
\hline B4090 & $0 \cdot 047$ & $239 / 95$ & $>256$ & NA & $\mathrm{His}^{552} \rightarrow \mathrm{Tyr}$ & $\mathrm{B}: \mathrm{NT}: \mathrm{P} 1.2$ \\
\hline B4100 & 019 & $256 / 95$ & $>256$ & NA & $\mathrm{His}^{552} \rightarrow \mathrm{Tyr}$ & B : $15:$ P1.6 \\
\hline B4134 & $0 \cdot 25$ & $298 / 95$ & $>256$ & NA & $\mathrm{Ser}^{548} \rightarrow$ Phe & B : $4:$ P1.9 \\
\hline B4135 & $0 \cdot 25$ & $299 / 95$ & 8 & 5 & $\mathrm{Ser}^{600} \rightarrow$ Leu & $\mathrm{B}: 22: \mathrm{P} 1.4$ \\
\hline C4137 & $0 \cdot 125$ & $301 / 95$ & $>256$ & 4 & $\mathrm{His}^{552} \rightarrow$ Asn & $\mathrm{C}: 2 \mathrm{~b}: \mathrm{P} 1.2$ \\
\hline W4175 & $0 \cdot 38$ & $348 / 95$ & $>256$ & 6 & $\mathrm{Ser}^{600} \rightarrow$ Leu & $\mathrm{W}_{135}: \mathrm{NT}: \mathrm{P} 1.2 .5$ \\
\hline B4241 & $0 \cdot 5$ & $71 / 96$ & $>256$ & 8 & $\begin{array}{l}\mathrm{His}^{552} \rightarrow \text { Asn; } \\
\quad \mathrm{Ala}^{558} \rightarrow \mathrm{Val}\end{array}$ & $\mathrm{B}: \mathrm{NT}: \mathrm{NST}$ \\
\hline B4260 & $0 \cdot 25$ & $93 / 96$ & 24 & 5 & $\mathrm{Ser}^{600} \rightarrow$ Leu & $\mathrm{B}: 22: \mathrm{P} 1.15$ \\
\hline C4267 & $0 \cdot 5$ & $100 / 96$ & 24 & 7 & $\mathrm{Ser}^{526} \rightarrow \mathrm{Ala}$ & $\mathrm{C}: 2 \mathrm{~b}: \mathrm{P} 1.2 .5$ \\
\hline C4337 & $0 \cdot 38$ & $184 / 96$ & $>256$ & 4 & $\mathrm{His}^{552} \rightarrow \mathrm{Tyr}$ & $\mathrm{C}: 2 \mathrm{~b}: \mathrm{P} 1.10$ \\
\hline B4357 & $0 \cdot 38$ & $209 / 96$ & $>256$ & 6 & $\mathrm{His}^{552} \rightarrow \mathrm{Tyr}$ & $\mathrm{B}: \mathrm{NT}: \mathrm{P} 1.14$ \\
\hline B4365 & $0 \cdot 38$ & $216 / 96$ & $>256$ & 3 & $\mathrm{His}^{552} \rightarrow \mathrm{Tyr}$ & $\mathrm{B}: 4: \mathrm{P} 1.14$ \\
\hline W4966 & $>256$ & $418 / 97$ & $>256$ & 3 & $\mathrm{His}^{552} \rightarrow \mathrm{Tyr}$ & $\mathrm{W}_{135}: 2 \mathrm{a}: \mathrm{P} 1.2 .5$ \\
\hline B5253 & $0 \cdot 125$ & $750 / 97$ & 8 & NA & $\mathrm{Ser}^{534} \rightarrow$ Pro & $\mathrm{B}: \mathrm{NT}: \mathrm{NST}$ \\
\hline B5648 & 1 & $431 / 98$ & $>256$ & 5 & $\mathrm{His}^{552} \rightarrow \mathrm{Tyr}$ & $B: 22:$ NST \\
\hline B5724 & 24 & $523 / 98$ & $>256$ & 4 & $\mathrm{His}^{552} \rightarrow \mathrm{Tyr}$ & $\mathrm{B}: 22: \mathrm{P} 1.5$ \\
\hline B5726 & $>256$ & $525 / 98$ & $>256$ & 4 & $\mathrm{His}^{552} \rightarrow \mathrm{Tyr}$ & $\mathrm{B}: 22: \mathrm{P} 1.5$ \\
\hline
\end{tabular}

${ }^{*}$ Number of passages after which final MIC was observed; NA, data not available.

(primers U3/U5; Table 1, Fig. 1) from strain 93/96 (MIC $24 \mu \mathrm{g} \mathrm{ml}^{-1}$ ). The amplified sequence harboured the meningococcal uptake sequence (5'-GCCGTCTGAA; Goodman \& Scocca, 1988, 1991) close to its 5' end.

Strain LB2927, isolated from a case of invasive meningococcal disease, was used as a recipient strain (MIC for rifampicin: 0.047 $\mu \mathrm{g} \mathrm{ml}^{-1}$ ). Transformation of LB2927 with PCR amplicons of type A was done as described elsewhere (van der Ley \& Poolman, 1992) with modifications. Briefly, starting from a pure culture, meningococci were grown in $3 \mathrm{ml}$ standard cell culture medium RPMI 1640 (Gibco-BRL) supplemented with $10 \%$ fetal calf serum to mid-exponential phase. The meningococci were spun down and resuspended in $100 \mu \mathrm{RPMI} 1640$. DNA was added to the bacterial suspension to a final concentration of $3 \mu \mathrm{g} \mathrm{ml}^{-1}$ with and without $\mathrm{MgCl}_{2}$ at a final concentration of $1 \mathrm{mM}$, and the mixture was then subjected to a $42{ }^{\circ} \mathrm{C}$ heat-shock in a water bath for exactly $45 \mathrm{~s}$. The cultures were incubated overnight in order to enable recombination and to ensure phenotypic expression of the integrated DNA. Transformed meningococcal cultures were streaked onto freshly prepared GC agar (chocolate agar supplemented with IsoVitalex) containing either 32 or $256 \mu \mathrm{g}$ rifampicin $\mathrm{ml}^{-1}$ (GC agar produced in-house, rifampicin from Sigma) as well as on GC agar without antibiotic (growth control).

All colonies that were obtained after transformation on rifampicin-agar plates were used in a simple assay to discriminate between transformed meningococci and bacteria that had become resistant through spontaneous point mutations. Briefly, the PCR amplicon used for transformation displayed five sites specific for the restriction enzyme HpyCH4III and the corresponding sequence of the acceptor strain harboured only four sites (Fig. 1). Subgenic fragment A (1352 bp) covering these sites was amplified by adding a toothpick of bacterial growth to PCR mixtures. Following amplification, the DNA was digested using $2 \mathrm{U}$ HpyCH4III (New England Biolabs). The banding patterns were checked on $2 \%$ agarose gels. A pattern of five bands (of 577, 398, 206, 137 and 34 bp) indicated that a grown colony was resistant due to spontaneous mutation; bands of $547,398,206,137,34$ and 30 bp indicated that a colony represented a colony grown from transformed meningococci. Transformants and 12 randomly selected spontaneously mutated isolates were analysed further by sequencing.

\section{RESULTS}

Fourteen meningococcal strains were selected in a stepwise manner towards rifampicin resistance. The majority of the resulting resistant strains displayed final MICs of $>256 \mu \mathrm{g}$ $\mathrm{ml}^{-1}$ (mean number of $5.43 \pm 0.51$ subcultures in order to achieve resistance). Sequence analysis of the $790 \mathrm{bp}$ subgenic $r p o B$ fragment (fragment $B$ ) derived from each of the strain pairs (i.e. the original susceptible strain and the corresponding fully resistant one) yielded only a single nucleotide difference within the region sequenced. The only exception was the strain pair B4241 and 71/96, which differed by two nucleotides. Analysis of the deduced amino acid sequences showed that each of these point mutations lead to amino acid substitutions, which are given in Table 1. The most frequent amino acid substitution observed in our strain collection was the change at $\mathrm{His}^{552}$, which has been reported previously (Carter et al., 1994; Nolte, 1997; Stefanelli et al., 2001). In addition, four hitherto-undescribed amino acid substitu- 


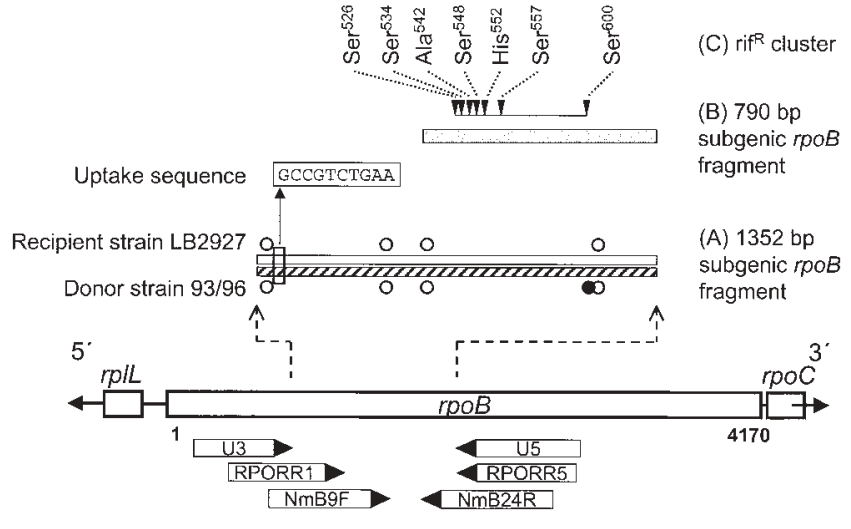

Fig. 1. Schematic representation of the rpoB gene within the $r$ r IKAJL-rpoBC operon. The position of the primers used for amplification and/or sequencing is given (Table 2). The flanking genes rpoC and $\mathrm{rp} / \mathrm{L}$ are not given to scale. Enlarged is the $1352 \mathrm{bp}$ amplicon (fragment $A$ ), derived by using primers $U 3$ and $U 5$, shown as an open bar for the original rifampicin-susceptible recipient strain and as a hatched bar for the resistant donor strain. Restriction sites for $\mathrm{HpyCH} 4 \mathrm{III}$ are shown as open circles; a fifth site, characterizing the donor strain, is shown as a filled circle. The position of the meningococcal uptake sequence is indicated by a box. The shaded bar shows the $790 \mathrm{bp}$ fragment (B) amplified using RPORR1 and RPORR5, spanning the cluster of rifampicin resistance-conferring mutations (rif ${ }^{\mathrm{R}}$ cluster; $\mathrm{C}$ ). Previously known mutations are indicated by black triangles (data combined from this paper and from Carter et al., 1994; Nolte, 1997; Stefanelli et al.,2001); the mutation leading to a substitution at $\mathrm{Ala}^{558}$ is not shown as this mutation does not confer resistance. Position $\mathrm{His}^{552}$ is a hot spot; three different mutations have been described to date.

tions were found in six strains. Three strains (MIC 8, 16 and $>256 \mu \mathrm{g} \mathrm{ml}^{-1}$ ) displayed a substitution of Ser ${ }^{600}$ by Leu, one strain displayed a change from $\mathrm{Ser}^{534}$ to Pro (MIC $8 \mu \mathrm{g} \mathrm{ml}^{-1}$ ) and another strain a change at position 526, where Ser was changed to Ala (MIC $24 \mu \mathrm{g} \mathrm{ml}^{-1}$ ). Finally, we observed a mutation leading to a change at position $\mathrm{Ala}^{558}$ to Val, which occurred as a second substitution besides the well-known His $^{552}$ in strain 71/96. All mutations, including those that have been published previously, are shown in Fig. 1.

\section{Transformation}

In order to demonstrate that mutations observed after stepwise selection are responsible for acquired resistance, PCR-amplified subgenic 1352 bp fragments (fragment A in Fig. 1) harbouring the mutation conferring the amino acid substitution Ser ${ }^{600}$ were used for transformation experiments. The DNA sequence of the amplicon of the donor strain 93/96 (MIC $24 \mu \mathrm{g} \mathrm{ml} \mathrm{m}^{-1}$ ) was identical with the sequence of another strain (299/95) found to display an MIC of about $8 \mu \mathrm{g} \mathrm{ml}^{-1}$ (Table 1). The deduced amino acid sequence of the DNA fragment used for transformation was identical in strain 299/95 (MIC $8 \mu \mathrm{g} \mathrm{ml}^{-1}$ ) and strain 348/95 (MIC $256 \mu \mathrm{g} \mathrm{ml}^{-1}$ ).

The 1352 bp fragment of the donor strain harboured five sites for the restriction enzyme $H p y C H 4 I I I$, whereas the corresponding fragment of the susceptible recipient harboured only four sites (Fig. 1), resulting in different RFLP patterns following digestion of the amplified fragment A. In addition, the subgenic fragment A of the recipient strain LB2927 differed from the respective fragment of the resistant donor strain by a number of silent mutations (genetic marker), which are described in detail by Nolte (1997). In brief, the donor strain belonged to sequence type 2 in that paper, characterized by three consecutive triplets each mutated in the third position $(\mathrm{C} \rightarrow \mathrm{A}$ at position $1707, \mathrm{C} \rightarrow \mathrm{A}$ at position 1710 and $\mathrm{C} \rightarrow \mathrm{T}$ at position 1713 of the gene) not present in the recipient strain, which belonged to sequence type 1 .

Following four independent transformations, 198 resistant colonies were observed (80 on agar plates containing $32 \mu \mathrm{g}$

Table 2. Primers used in this study

All primers except NmB9F and NmB24R, which were sequencing primers only, and U5, which was used for amplification only, were used for amplification and sequencing. Primer sequences were obtained from the sequence of the entire $r p o B$ gene of N. meningitidis (EMBL/GenBank/DDBJ accession no. Z54353; O. Nolte, unpublished). $T_{\mathrm{m}}$ values were supplied by the manufacturer of the oligonucleotides (TIBMolBiol); for amplification, the annealing temperature was set to $2{ }^{\circ} \mathrm{C}$ below the given $T_{\mathrm{m}}$. NA, Not applicable.

\begin{tabular}{|llllc|}
\hline Primer & \multicolumn{1}{c}{ Sequence $\left(\mathbf{5}^{\prime} \rightarrow \mathbf{3}^{\prime}\right)$} & Orientation & $\mathbf{T}_{\mathbf{m}}\left({ }^{\circ} \mathbf{C}\right)$ & $\begin{array}{c}\text { Amplicon } \\
\text { size }(\mathbf{b p})\end{array}$ \\
\hline U3 & CTACGACAAAGAAACGTTCTATTT & Sense & $53 \cdot 2$ & 1153 \\
U5 & ATATATTGGACGCGGTTCCGGGCGTT & Antisense & $79 \cdot 5$ & \\
RPORR1 & AAAAACTGTCCGAAGCCCAACAAAACTCT & Sense & $67 \cdot 2$ & 790 \\
RPORR5 & ATATATTGGACGCGGTTCCGGGCGTT & Antisense & $70 \cdot 6$ & \\
NmB9F & ATGATTAATGCAAAACCTGT & Sense & $49 \cdot 6$ & NA \\
NmB24R & TTCGGACCTTCAGGCGTTT & Antisense & $59 \cdot 5$ & \\
MTR6 & TCGCAGGAAGAGAACCAC & Sense & $53 \cdot 5$ & $980^{*}$ \\
MTRC1rev & CATCGCCTTAGAAGCATAA & Antisense & $49 \cdot 9$ & \\
\hline
\end{tabular}

${ }^{\star}$ Amplicon of $980 \mathrm{bp}$ if a $158 \mathrm{bp}$ insertion in the $m t r R$ promoter region is present (Abadi et al., 1996). 
rifampicin $\mathrm{ml}^{-1}$ and 118 on plates containing $256 \mu \mathrm{g} \mathrm{ml}^{-1}$ ). Colonies resistant through spontaneous mutations were observed exclusively after transformation without the addition of $\mathrm{MgCl}_{2}$. Only three resistant colonies could be identified by their HpyCH4III restriction fragment pattern at $r p o B$ as transformants, all of which grew after transformation in the presence of $1 \mathrm{mM} \mathrm{MgCl}$. Two of the three transformants were undoubtedly confirmed following sequence analysis of the $1352 \mathrm{bp}$ fragment: (i) the Ser ${ }^{600}$-conferring mutation was present and (ii) the genetic marker, absent in the original recipient strain, was present in both recipient strains after transformation. The third transformant, however, harboured the genetic marker sequence of the donor strain but did not display the $\operatorname{Ser}^{600}$ mutation. Rather, the mutation in codon 552 (nucleotide position 1654) was found, leading to a substitution of His by Tyr in the deduced amino acid sequence.

Of the 195 colonies found to be resistant due to spontaneous mutations, 12 were selected randomly for sequence determination of the $1352 \mathrm{bp}$ subgenic fragment A. All of the sequences were identical and did not contain the genetic marker. All 12 strains, however, were found to have acquired resistance due the mutation $\mathrm{C} \rightarrow \mathrm{T}$ at position 1654 , leading to the resistance-conferring mutation at amino acid position $\mathrm{His}^{552}$ of the protein.

The overall mutation frequency leading to spontaneous mutants was calculated to be $2.59 \times 10^{-7}$ and the transformation efficiency was determined to be $1.93 \times 10^{-9}$.

The transformed meningococci, as well as the spontaneously mutated strains, grew with comparable efficacy on plates containing both 32 and $256 \mu \mathrm{g}$ rifampicin $\mathrm{ml}^{-1}$.

\section{DISCUSSION}

The results described here indicate that the molecular mechanism of acquisition of rifampicin resistance by meningococci is more complicated than was known hitherto. The description of four new mutations, all observed in strains selected in vitro rather than in naturally occurring strains, that have not to our knowledge been reported previously for $N$. meningitidis raises the total number of known mutations to ten. In addition, the strains examined here displayed various levels of resistance ranging from 8 to over $256 \mu \mathrm{g}$ $\mathrm{ml}^{-1}$. However, although the strains were selected stepwise, all but one of the resistant strains were found to have only one altered amino acid site within the $r p o B$ fragment analysed. This result agrees with the observation that, although a stepwise increase in MIC was observed, resistance was acquired within one step once the strains under examination exceeded an MIC of $4 \mu \mathrm{g} \mathrm{ml}{ }^{-1}$.

It was discussed before that particularly high-level rifampicin resistance may be acquired by a point mutation in $r p o B$ and by another, still-uncharacterized mechanism(s) (Abadi et al., 1996). However, we did not find alterations in the $m t r R$ promoter region when checking for the presence of an $158 \mathrm{bp}$ insertion (results not shown). It could be questioned that only a fragment of $r p o B$ was sequenced, leaving the possible occurrence of other mutations that we did not identify. Indeed, Jin \& Gross (1988) described 19 different mutations that conferred resistance among 45 rifampicin-resistant strains of E. coli. Amongst the mutations described, two are outside the region sequenced so far in meningococci. This means that, in theory, more than the particular point mutations that we have detected could have conferred resistance by means of a cumulative mechanism. To rule out the contribution of other undetected mutations to rifampicin resistance in the strains studied, a PCR-amplified subgenic $r p o B$ fragment harbouring the $\operatorname{Ser}^{600}$ mutation, which was found in strains displaying different MICs, was transformed into a wild-type recipient $N$. meningitidis strain. The resulting transformants were found to be resistant to levels of $256 \mu \mathrm{g}$ rifampicin $\mathrm{ml}^{-1}$. These results suggest that high-level resistance can be transferred by a single point mutation into a susceptible acceptor strain. However, transformation rates in our experiments were extremely low. This may be explained because we used PCR amplicons rather than genomic DNA for transformation.

A number of the spontaneous mutant strains were also sequenced at the $r p o B$ locus in order to describe their resistance mechanisms. Interestingly, all 12 isolates studied displayed the mutation at position 1656 , leading to a substitution at amino acid position 552, known to be the most frequent described substitution in rifampicin-resistant meningococci. This mutation seems therefore to be a hot spot in the subgenic region of $r p o B$, both in resistant strains from the field as well as in experimental strains. Spontaneous mutants were observed on plates containing both 32 and $256 \mu \mathrm{g}$ rifampicin $\mathrm{ml}^{-1}$.

In summary, we have described new mutations that lead to rifampicin resistance in meningococci, but we did not find evidence for more than a one-step mechanism of acquisition of resistance. Our data cannot explain the presence of different levels of resistance. For instance, the newly described mutation $\mathrm{Ser}^{600} \rightarrow$ Leu was found in three strains that displayed MICs of about 8, 16 and $>256 \mu \mathrm{g} \mathrm{ml}{ }^{-1}$. Transformed meningococci, having adopted the $\operatorname{Ser}^{600}$ mutation, however, were found to be of the high-level resistance phenotype. It should be considered that a phenotypic adaptation confers high-level resistance to rifampicin rather than additional mutations or additional genetic mechanisms acting synergistically with the $r p o B$ mutations described already.

\section{ACKNOWLEDGEMENTS}

The results described in this paper were presented in part at the 51st Conference at the Deutsche Gesellschaft für Hygiene \& Mikrobiologie, 30 September-4 October 2001, Aachen, Germany.

\section{REFERENCES}

Abadi, F. J. R., Carter, P. E., Cash, P. \& Pennington, T. H. (1996). Rifampin resistance in Neisseria meningitidis due to alterations in membrane permeability. Antimicrob Agents Chemother 40, 646-651. 
Aubry-Damon, H., Soussy, C.-J. \& Courvalin, P. (1998). Characterization of mutations in the $r p o B$ gene that confer rifampin resistance in Staphylococcus aureus. Antimicrob Agents Chemother 42, 2590-2594.

Carter, P. E., Abadi, F. J. R., Yakubu, D. E. \& Pennington, T. H. (1994). Molecular characterization of rifampin-resistant Neisseria meningitidis. Antimicrob Agents Chemother 38, 1256-1261.

Goodman, S. D. \& Scocca, J. J. (1988). Identification and arrangement of the DNA sequence recognized in specific transformation of Neisseria gonorrhoeae. Proc Natl Acad Sci U S A 85, 6982-6986.

Goodman, S. D. \& Scocca, J. J. (1991). Factors influencing the specific interaction of Neisseria gonorrhoeae with transforming DNA.J Bacteriol 173, 5921-5923.

Hagman, K. E., Pan, W., Spratt, B. G., Balthazar, J. T., Judd, R. C. \& Shafer, W. M. (1995). Resistance of Neisseria gonorrhoeae to antimicrobial hydrophobic agents is modulated by the mtrRCDE efflux system. Microbiology 141, 611-622.

Jin, D. J. \& Gross, C. A. (1988). Mapping and sequencing of mutations in the Escherichia coli rpoB gene that lead to rifampicin resistance. $J \mathrm{Mol}$ Biol 202, 45-58.
Nolte, O. (1997). Rifampicin resistance in Neisseria meningitidis: evidence from a study of sibling strains, description of new mutations and notes on population genetics. J Antimicrob Chemother 39, 747-755.

Pan, W. \& Spratt, B. G. (1994). Regulation of the permeability of the gonococcal cell envelope by the mtr system. Mol Microbiol 11, 769-775.

Stefanelli, P., Fazio, C., La Rosa, G., Marianelli, C., Muscillo, M. \& Mastrantonio, P. (2001). Rifampicin-resistant meningococci causing invasive disease: detection of point mutations in the $r p o B$ gene and molecular characterization of the strains. J Antimicrob Chemother 47, 219-222.

Telenti, A., Imboden, P., Marchesi, F., Lowrie, D., Cole, S., Colston, M. J., Matter, L., Schopfer, K. \& Bodmer, T. (1993). Detection of rifampicinresistance mutations in Mycobacterium tuberculosis. Lancet 341, $647-650$.

van der Ley, P. \& Poolman, J. T. (1992). Construction of a multivalent meningococcal vaccine strain based on the class 1 outer membrane protein. Infect Immun 60, 3156-3161. 